\title{
News of Effective Temperatures of L Dwarfs
}

\author{
Andreas Schweitzer ${ }^{1}$, John E. Gizis ${ }^{2}$, France Allard ${ }^{3}$, Peter H. \\ Hauschildt ${ }^{1}$ \\ ${ }^{1}$ Dept. of Physics \& Astronomy, Univ. of Georgia, Athens, GA 30602; \\ ${ }^{2}$ Dept. of Physics \& Astronomy, Univ. of Delaware, Newark, DE \\ 19716; ${ }^{3}$ CRAL, ENS Lyon, 69364, France
}

\begin{abstract}
In this work we report recent spectral analyses of $\mathrm{L}$ dwarfs and our success in measuring $\mathrm{T}_{\text {eff }}$ and $\log (\mathrm{g})$. Using dust filled atmospheres for early $\mathrm{L}$ dwarfs and rained out atmospheres for late $\mathrm{L}$ dwarfs we could derive $\mathrm{T}_{\text {eff }}$ of 1400 to $2000 \mathrm{~K}$ for L8 to M9.5 dwarfs respectively. We also give an outlook what we can achieve with future models that are improving the fits to intermediate $L$ dwarfs and IR spectra.
\end{abstract}

\section{Analysis of $L$ dwarfs}

In two recent papers (Schweitzer et al. 2001, 2002) we performed a detailed multiwavelength spectroscopic study of $L$ dwarfs. We used optical low resolution spectra from Kirkpatrick et al. (2000, and references therein), high resolution

Table 1. The derived values for $\mathrm{T}_{\text {eff }}$ and $\log (\mathrm{g})$ for the sample.*

\begin{tabular}{lllrr}
\hline Name & & Spectral Type & $\mathrm{T}_{\text {eff }}$ & $\log (\mathrm{g})$ \\
\hline 2MASSW & $\mathrm{J} 0149090+295613$ & $\mathrm{M} 9.5$ & $2100 \mathrm{~K}$ & 6.0 \\
2MASS & $\mathrm{J} 2234139+235956$ & $\mathrm{M} 9.5$ & $2000 \mathrm{~K}$ & 6.0 \\
2MASP & $\mathrm{J} 0345432+254023$ & $\mathrm{~L} 0$ & $1900 \mathrm{~K}$ & 6.0 \\
2MASSW & $\mathrm{J} 0147334+345311$ & $\mathrm{~L} 0.5$ & $1900 \mathrm{~K}$ & 6.0 \\
2MASSI & $\mathrm{J} 0746425+200032$ & $\mathrm{~L} 0.5$ & $2000 \mathrm{~K}$ & 6.0 \\
2MASSW & $\mathrm{J} 1439284+192915$ & $\mathrm{~L} 1$ & $1900 \mathrm{~K}$ & 6.0 \\
2MASSI & $\mathrm{J} 1726000+153819$ & $\mathrm{~L} 2$ & $1900 \mathrm{~K}$ & 6.0 \\
2MASSW & $\mathrm{J} 1146345+223053$ & $\mathrm{~L} 3$ & $1800 \mathrm{~K}$ & 5.0 \\
2MASSW & $\mathrm{J} 0036159+182110$ & $\mathrm{~L} 3.5$ & $1800 \mathrm{~K}$ & 5.5 \\
2MASS & $\mathrm{J} 0850359+105716$ & $\mathrm{~L} 6$ & $1700 \mathrm{~K}$ & 5.5 \\
2MASSW & $\mathrm{J} 0920122+351742$ & $\mathrm{~L} 6.5$ & $1700 \mathrm{~K}$ & 6.0 \\
2MASSI & J0825196+211552 & $\mathrm{L} 7$ & $1700 \mathrm{~K}$ & 6.0 \\
2MASSW & $\mathrm{J} 0929336+342952$ & $\mathrm{~L} 7.5$ & $1400 \mathrm{~K}$ & 5.0 \\
2MASSI & $\mathrm{J} 0328426+230205$ & $\mathrm{~L} 8$ & $1400 \mathrm{~K}$ & 6.0 \\
2MASSW & $\mathrm{J} 1632291+190441$ & $\mathrm{~L} 8$ & $1400 \mathrm{~K}$ & 5.5 \\
2MASSW & J1523226+301456 & $\mathrm{L} 8$ & $1400 \mathrm{~K}$ & 5.5 \\
\hline \hline
\end{tabular}

*We used AMES-Dusty models for the top eight objects, AMES-Cond models for the bottom seven objects and both for the L3.5. 

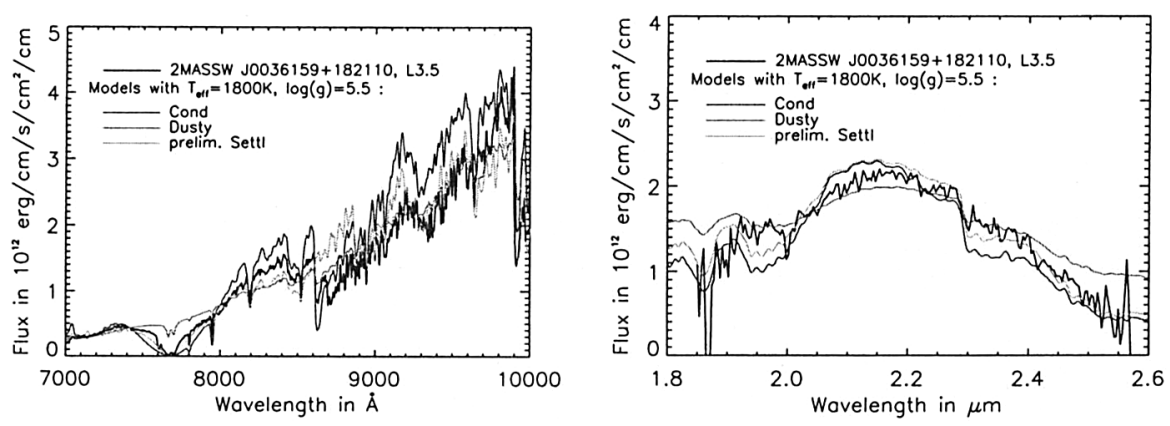

Figure 1. Comparison of the best fitting Dusty and Cond models for the L3.5 dwarf with a preliminary model that accounts for settling.

spectra obtained with HIRES on Keck I (see Schweitzer et al. 2001, for details) and K band spectra obtained with CRSP (see Schweitzer et al. 2002, for details). We used our then latest models to perform the analysis. A detailed description of the physics can be found in Allard et al. (2001). The most important features are line lists for all important molecules, dust formation in equilibrium inside the photosphere and dust opacities due to 40 dust species. For the two previous papers the models were available in two limiting cases: AMES-Dusty and AMESCond. For the AMES-Dusty models the dust stays in the layer in which it forms. This effectively adds maximum dust opacity and fits early L dwarfs (Tab. (1)). For the AMES-Cond models the dust rains out completely. This effectively removes the fraction of elements bound in dust from the atmosphere and fits late L dwarfs. None fit intermediate L dwarfs and IR spectra consistently.

\section{Outlook}

We are currently calculating newer models which calculate self consistent rain out of dust. These models are producing a dust stratification depending on stellar parameters where the settling of grains is counter-balanced by convective mixing into the upper layers of the photosphere. Although we could not do a detailed fitting with different models, we find that the same parameters now seem to be in good agreement with both optical and infrared spectra (Fig. (1)).

\section{References}

Allard, F., Hauschildt, P. H., Alexander, D. R., Tamanai, A., \& Schweitzer, A. 2001, ApJ, 556, 357

Kirkpatrick, J. D., Reid, I. N., Liebert, J., Gizis, J. E., Burgasser, A. J., Monet, D. G., Dahn, C. C., Nelson, B., et al., 2000, AJ, 120, 447

Schweitzer, A., Gizis, J. E., Hauschildt, P. H., Allard, F., Howard, E. M., \& Kirkpatrick, J. D. 2002, ApJ, 566, 435

Schweitzer, A., Gizis, J. E., Hauschildt, P. H., Allard, F., \& Reid, N. 2001, ApJ, 555,368 